\title{
Time and Accuracy Analysis of Skew Detection Methods for Document Images
}

\author{
Sunita Mehta \\ Chandigarh College of Engineering and Technology, U.T., Chandigarh, India \\ E-mail:sunitavatta@gmail.com \\ *Ekta Walia \\ Department of Computer Science, University of Saskatchewan, Saskatoon, Canada \\ E-mail: wekta@yahoo.com \\ Maitreyee Dutta \\ Computer Science Department, NITTTR, Chandigarh, India \\ E-mail: d_maitreyee@yahoo.co.in
}

\begin{abstract}
Detecting skew angle in a document image has been an area of research interest for a long time. This paper presents an experimental analysis of various existing skew detection techniques involving methods such as Radon transform, Hough transform, Principal Component Analysis (PCA), PCA with Wavelet transform and Moments with Wavelet transform. Detailed analysis of existing skew detection method against the parameters time complexity, space complexity, robustness, accuracy, flexibility, etc. has been carried out for seven different categories of digital documents. The categories of these documents spans from those containing handwritten text in different languages, to the ones with both text and pictures. Radon transform is observed to be the fastest method when the image size is small and works with virtually all types of documents. It is an accurate method as well as works faster, even with the document containing pictures. PCA method is also faster than Hough transform for machine printed documents but used less for real time skew distortion due to its limitations. If the document image size is large, then Moments with Wavelet transform has better time complexity than other methods, but do not work well with documents containing images. Hough transform is the most accurate method, though it is computationally expensive.
\end{abstract}

Index Terms-Document Image Processing, Skew detection, Skew correction, Discrete Wavelet transform, Principal Component Analysis, Hough transform, Radon transform, Moments.

\section{INTRODUCTION}

Document image processing (DIP) has turned out to be an essential technology in the digitization of office documentation tasks. Earlier paper documents were used to store large amount of information, but now due to the advent in technology, document can now be stored electronically using the document processing techniques consisting of three steps namely scanning, digitization and conversion of scanned images into machine editable forms. When we scan or copy documents, there are chances of document skew distortion error. The direction of text line determines the skew angle. If the lines are aligned horizontally or vertically, depending upon the language, it means that there is no skew in a document image. Due to skew distortion, difficulty arises in document analysis, document understanding, document image mosaicing, character segmentation and identification. Consequently, detecting the skew of a document image and correcting it are substantial problems in realizing a practical document reader.

Optical Character Recognition (OCR) helps to translate various categories of documents which include PDF files, scanned paper documents or images taken by a digital camera into a form which can be processed and changed as per requirement. Virtually all of the Optical Character Recognition systems are very responsive to skew in text document images. It is not possible to get a hundred percent accuracy with the methods of OCR systems due to the problem of skew angle and errors in noise removal methods.

The document skew can be classified as Global or Local. In case of global skews, all the text lines of a document have the same orientation, whereas in case of local skew each line can be skewed at dissimilar angle.

Chandan Singh, Nitin Bhatia and Amandeep Kaur "Ref. [1]" introduced a skew detection and correction algorithm which are further classified into three parts: a preprocessing stage, which makes use of a simplified form of a black adjacency graph (BAG), Hough Transform (HT) which makes use of the voting process and using the rotation method for the purpose of deskewing of the image. Though this method reduces the time complexity, but the drawback of this method is that it does not provide suitable results for Indian scripts 
where the headline is a part of the script. They have implemented skew correction using both the approaches, i.e. forward rotation and inverse rotation. The speed offorward rotation is more than the inverse rotation. The main disadvantage of the forward rotation is that it generates an image with various unwanted white pixels within the body of a character which degrades the overall performance of an OCR system.

N. Nandini, K. Srikanta Murthy, and G. Hemantha Kumar "Ref. [2]", A. Amin and S. Fischer "Ref. [3]", Stuart C.Hinds "Ref. [4]", S. N. Srihari and V. Govindraju "Ref. [5]" and V. N. Manjunath Aradhya "Ref. [6]" have also used Hough Transform to determine the skew angle.

Rajiv Kapoor, Deepak Bagai and T.S.Kamal "Ref. [9]" explained two methods. The first one is used to detect skew of words and the second one correct skews from handwritten words. They have used Radon Transform based projection profile technique for the implementation of skew detection and removal method. The advantage of this method is that it does not need pre-processing and it works similarly fine even in the presence of noise. The disadvantage is that problem may still come in the case of words with non-uniform Shiro-Rekha or characters without Shiro-Rekha.

S. Mehta, E. Walia, and M. Dutta "Ref. [14]" discussed skew detection method based on Moments and Wavelet transforms. The only drawback is that it does not work with pictures document.

C. N. Paunwala, S. Patnaik, and M. Chaudhary "Ref. [15]" described an efficient approach for skew correction of license plate based on Wavelet Transform and Principal Component Analysis. The basic concept of PCA is to lessen the dimensionality of a data set by considering a large number of interrelated variables while preserving as much as possible of variation present in the data set. The advantage of this method is that it is simple and has a reduced amount of computational complexity. The drawback is this method doesn't work with picture documents.

L.Shutao, S.Qinghua, and S.Jun "Ref. [16]" discussed skew detection method using Wavelet Transform (WT) and Principal Component Analysis however the problem is that it doesn't work with picture documents.

Bishakha and Mrinaljit "Ref. [17]" suggested method which is used to detect the skew angle for Assamese language using vertical and horizontal projection profile method. In case of noisy images, horizontal projection method gives more accurate results. Also the time taken by the horizontal projection profile method is less than the vertical projection profile method. Drawbacks of these methods are that they are computationally expensive and can find a skew angle till $15^{\circ}$. Also, these methods do not work with more noisy images and broken characters.

D.Brodic, C.A.Maluckov, and L.Peng "Ref. [18]" implemented skew detection method for old printed documents by using connected component.

Shivakumara, G.Hemantha Kumar, and H.S.Varsha, "Ref. [19]" proposed technique based on boundary growing approach and Moments based method. This method is robust as it works with documents containing different languages and different fonts. But it fails for a document containing picture along with text.

Mandip Kaur and Simpel Jindal "Ref. [20]" proposed skew detection method using Fast Fourier Transform (FFT) technique. They have reduced document image size by applying DCT compression.

Deepak and Dalwinder "Ref. [21]" proposed approach which makes use of the Hough Transform, as this method is simple and accurate. But as we know that Hough Transform method takes more time so they have improved this method to reduce the time complexity without losing its accuracy. Also skew is corrected using forward rotation as it takes less time.

Sepideh Barekat Rezaei, Abdolhossein Sarrafzadeh and Jamshid Shanbehzadeh "Ref. [22]" surveyed various methods to detect the skew. These methods include Projection Profile Analysis, Hough Transform, Nearest Neighbor clustering, Cross-Correlation, Piecewise Painting algorithm, Piecewise covering by Parallelogram, Morphology. In some of the methods, the size of the image is reduced considering only the important features.

Khalil Ibrahim Alsaif and Montaha Tariq Alsarraj "Ref. [23]" demonstrated a novel approach based on Fan filter to determine the skew angle of a text image.

H. Yan "Ref. [24]" explained the method based on the interline cross-correlation in the document image. This method is quite time consuming for large images and the accuracy of the method is affected by the presence of graphics in the documents.

A.K. Das and B. Chanda "Ref. [25]" presented a novel method for automatic detection of skew in a document image using Mathematical Morphology. The proposed algorithm is tremendously fast as well as independent of script forms. The drawback of the method is its limited suitability for larger database.

Ruby and Ramandeep "Ref. [26]" presented discrete Fourier transform algorithm to compute the skew angle. The drawback is more time complexity.

Some common problems detected of existing methods "Ref. [1-6, 9-26]" from literature studied regarding skew angle detection of a document image are:

- Restriction on the detectable angle range.

- Restrictions on size or type of fonts.

- Depending on page layout. Usually the presence of graphics, borders, or tables constitutes a problem.

- A specific document resolution is required.

- High Computational Cost.

- Limitation to specific application.

- There are very few methods proposed to handle handwritten or mixed documents.

- $\quad$ Large text areas are required.

- Doesn't work with both types of skew i.e. global and local skew

In this paper, detailed analysis of existing skew angle detection methods against the parameters time complexity, space complexity, robustness, accuracy, 
flexibility, etc. for seven different categories of digital documents has been done.

Most of the skew distortion removal methods are based on following the techniques listed next.

\section{A. Hough transform}

The Hough transform provides a significant way out of the problems related with line and curve detection in a binary image. The basic method comprises of mapping points in the Cartesian space to sinusoidal curves in (rho, theta) i.e. $(\rho, \theta)$ space via a simple transformation. Each time a sinusoidal curve intersects with another sinusoidal curve for a value of $\theta$, there is the likelihood that a line corresponding to that $(\rho, \theta)$ coordinate value is present in the original image. An accumulator array is used to count the number of intersections for various $\rho$ and $\theta$ values. The skew angle is then determined by the theta values corresponding to the highest number of counts in the accumulator array "Ref. [1, 2, 3, 4, 5, 6, 7, 8]".

\section{B. Projection profile}

The projection features are used to determine the skew angle "Ref. [8, 9, 10, 17]". In this technique, numbers of projections are acquired at different angles. Then, close to the best expected orientation and variations in the bin heights along the profile are observed for each projection. The skew angle is given by the maximum peak in the projection to best match to the text lines. The projection profile has maximum height peaks for text, since scan lines align with text lines at the correct skew angle.

\section{Fourier Method}

It is used to decompose an image into its sine and cosine components of different frequencies, each multiplied by a different coefficient "Ref. [8, 20]". The main characteristic of this method is that it allows us to work in the Fourier domain and then return to the original image without losing any kind of information. The angle of direction in which the density of Fourier space is large is considered to be the skew angle.

\section{Radon transform}

The Radon function computes projections of an image matrix along specified directions "Ref. [8, 9, 11]". A projection of a two dimensional function is a set of line integrals. The Radon function takes multiple, parallel beam projections of the image from different angles by rotating the source around the centre of the image. The procedure is repeated for all desired angle in the range $\left[0^{\circ}\right.$, $180^{\circ}$ ]. The slope determines the skew angle.

\section{E. Principal Component Analysis (PCA)}

PCA is a technique that can be used to simplify a dataset. It can be used for reducing dimensionality. As mentioned in "Ref. [12, 13]" for a given binary image, firstly black pixels of the image are mapped in a two dimensional vector. Then, the unit vector for the maximized projection profile deviation is obtained. This unit vector is called the Principal Component of the given vector set and it is equal to the Eigenvector that is associated with the largest absolute Eigen perpendicular value of this set. The skew angle is found using the direction of the Principal Component.

\section{F. Principal Component Analysis with Wavelet transform}

In this case, the size of the image is reduced using two dimensional Discrete Wavelet Transform (DWT) "Ref. [8]". After applying first level wavelet decomposition to skewed image, the image gets divided into four frequency bands resulting quarter size decomposition outputs, i.e. LL1 sub band corresponds to an approximation level, LH1 gives details in the horizontal direction, the HL1 sub band provides details in the vertical level and the $\mathrm{HH} 1$ sub band provides detail in the diagonal direction. The details of image after second level Wavelet decomposition i.e. LL1 gets further divided into four parts. In this case, that quarter size approximation sub image is then replaced by four more quarter size bands "Ref. [14]". Then the PCA method is applied to reduced image to determine the skew angle.

\section{G. Moments with Wavelet transform}

In this case "Ref. [14]" also, the size of the image is reduced using Wavelet transform as described in Section I.F. Then on reduced image, moments approach as mentioned below is used to determine the skew angle.

The moment of a two dimensional continuous function "Ref. [19]" of order $(m+n)$ is defined as

$$
\operatorname{Tmn}=\int_{-\infty}^{\infty} \int_{-\infty}^{\infty} x^{m} y^{n} f(x, y) d x d y
$$

$f(x, y)$ represents an image and $\mathrm{m}, \mathrm{n}=0,1,2 \ldots \ldots$ or continuous two dimensional image, equation of moments is given as below and for digital images $f(x, y)=1$

$$
\operatorname{Tmn}=\sum_{\mathrm{O}}^{i} \sum_{\mathrm{O}}^{i} x^{m} y^{n} f(x, y)
$$

Here image taken is converted into a square image of size $i \mathrm{x} i$ and $\mathrm{m}, \mathrm{n}=0,1 \ldots$

Central moments "Ref. [14, 19]" are defined by

$$
\mu_{m n}=\int_{-\infty}^{\infty} \int_{-\infty}^{\infty}(x-\bar{x})^{m}(y-\bar{y})^{n} f(x, y) d x d y
$$

Where $\bar{x}=\frac{T_{10}}{T_{00}}$ and $\bar{y}=\frac{T_{01}}{T_{00}}$ are components of the centroid.

For digital image $f(x, y)=1$, “(3), ” becomes

$$
\mu_{m n}=\sum_{0}^{i} \sum_{0}^{i}(x-\bar{x})^{m}(y-\bar{y})^{n}
$$

For the purpose of calculating the skew angle, the proposed algorithm uses second order central moments.

By substituting “(2)," into “(4),”, we can obtain “(5),", “(6),", "(7)," can be obtained. 


$$
\mu_{20}=T_{20}-\bar{x} T_{10}
$$

In the same manner, "(6)," and “(7)," can be obtained.

$$
\begin{gathered}
\mu_{11}=T_{11}-\bar{x} T_{01}=T_{11}-\bar{y} T_{01} \\
\mu_{02}=T_{02}-\bar{y} T_{01}
\end{gathered}
$$

Skew angle is the angle between the horizontal axis and the major axis of the object.

\section{METHODOLOGY}

The methods described in Section I are implemented through the following algorithms stated in this section.

\section{A. Skew detection using Hough transform}

This algorithm uses the Hough transform "Ref. [1, 2, 3 ]" to find the skew angle of document images. We have used Generalized Hough transform proposed by Duda and Hart "Ref. [7]". In this, firstly, Cartesian coordinates $(\mathrm{x}, \mathrm{y})$ are transferred into polar co-ordinates $(\rho, \theta)$. Hence, line equation in polar coordinates can be defined using these parameters. Here, $\rho$ represents the distance between the line connecting the polar coordinates to the origin where the $\mathrm{x}$-axis intersects the $\mathrm{y}$-axis and $\theta$ is the angle between $\mathrm{x}$-axis and $\rho$.

$$
y=\left(\frac{\cos \theta}{\sin \theta}\right) x+\left(\frac{p}{\sin \theta}\right)
$$

Which can be rearranged to

$$
\rho=x \cos \theta+y \sin \theta
$$

The algorithm to find skew is given below:

1. Read the image.

2. Binarize the image in case of scanned documents.

3. Detect the edges of the document using Canny filter.

4. Using Hough function of "(9)," find the Hough peaks.

5. Compute slope, which gives the skew angle in radians. Convert in degree to get the skew angle in degree.

\section{Advantages of Hough Transform}

- It is the most efficient algorithm to find a skew angle as it gives accurate results.

- It also works under noisy conditions.

Disadvantages of Hough Transform
- It is computationally very expensive.

- It is also not feasible for images whose layouts are complex because one has to first separate out the text regions in the document image.

\section{B. Skew detection using the Radon transform}

This algorithm uses Radon transform "Ref. [8, 9, 11]" to find the skew angle of document images.

Let $f(\boldsymbol{u})=f(u, v)$ be a continuous function. The Radon transform, $R f$ is a function defined on the space of straight lines $l$ by the line integral along each such line:

$$
R f(l)=\int_{l} f(u)|d u|
$$

Parameterization of any straight line $l$ with respect to arc length $t$ can be written

$$
(u(t), v(t))=((t \sin \theta+s \cos \theta),(-t \cos \theta+s \sin \theta))
$$

Radon transform can be expressed in these coordinates by

$$
\begin{aligned}
& R f(\theta, s)=\int_{-\infty}^{\infty} f(u(t), v(t)) d t \\
& =\int_{-\infty}^{\infty} f((t \sin \theta+s \cos \theta),((-t \cos \theta+s \sin \theta)) d t
\end{aligned}
$$

The algorithm to find skew is given below:

1. Read the image.

2. Binarize the image in case of scanned documents.

3. Find the edges of the document using the Sobel edge detection method "Ref. [8]".

4. Using Radon function, find the Radon transform for theta ranging from 0 to $179^{\circ}$ using "(12),".

5. Find the maximum of $R f$ and then find the slope of the corresponding column.

6. Slope gives the skew angle in radians. Convert in degree to get the skew angle in degree.

Advantages of Radon Transform

- The radon transform is suitable even in the presence of noise.

- Reduces time complexity of an algorithm

- Works with mixed type of document images

\section{Skew detection using Principal Component Analysis}

This algorithm uses PCA "Ref. [12, 13]" to find the skew angle of document images.

1. Read the image.

2. Binarize the image in case of scanned documents.

3. Map the black pixels of the image in twodimensional vectors.

4. For PCA to work properly, subtract the mean 
from each of the data dimensions. The mean subtracted is the average of intensities across each dimension.

5. The covariance matrix is then calculated as

$$
C V(X, Y)=\frac{1}{n-1} \sum_{i=1}^{n}\left(X_{i}-\bar{X}\right)\left(Y_{i}-\bar{Y}\right)^{\prime}
$$

Where $X$ and $Y$ denote the entire set of numbers in two different dimensions, $n$ is the number of elements in sets $X$ as well as $Y, X_{i}, Y_{i}$ are the $i^{\text {th }}$ number in the sets $X$ and $Y$ respectively, $\bar{X}$ and $\bar{Y}$ are the mean of the sets $X$ and $Y$. Calculate covariance matrix using "(13),".The result will be a square matrix.

6. Find the eigenvectors and eigenvalues of the covariance matrix. An eigenvector of a Square matrix $\mathrm{M}$ is a non-zero vector $\mathrm{V}$ that, when multiplied by $M$, yields the eigenvector multiplied by a single number $\delta$.

$$
\mathrm{MV}=\delta \mathrm{V}
$$

The number $\delta$ is called the eigenvalue of $M$ corresponding to $V$.

7. Take the eigenvector associated with the largest absolute eigenvalue.

8. Skew angle is found using the direction of the principal component, which is perpendicular to the direction of the text lines in the image.

Advantages of PCA

- It is efficient and one of the fastest algorithm to find skew angle.

Disadvantages of PCA

- It is not language independent means it doesn't detect the skew angle for all types of language.

- It doesn't work with images.

D. Skew Detection using Principal Component Analysis with Wavelet transform

This algorithm uses PCA with Wavelet Transform "Ref. $[14,15,16]$ " to find the skew angle of document images.

1. Convert the image to grayscale image, if not.

2. Decompose the image using 'Haar' wavelet. Four frequency sub bands, namely approximation level LL1, horizontal level LH1, vertical level HL1 and diagonal level HH1 are obtained. After second level wavelet decomposition, approximation subband gets further divided into four sub-bands LL2, LH2, HL2 and HH2. Consider LH1, HL1, LH2 and HL2 as they preserve horizontal and vertical details present in the image.

3. Use PCA method given in Section II.C to find a skew angle for LH1, HL1, LH2 and HL2 subbands.

4. Take the mean of all the angles, which give the final skew angle.

\section{Advantages of PCA with wavelet transform}

- In this method, the size of the original image is reduced, which improves time complexity.

- Well suited for large size images.

Disadvantages of PCA with wavelet transform

- This method doesn't work with image documents.

E. Skew Detection using Moments with Wavelet transform

The algorithm to determine the skew angle using Moments with Wavelet transform "Ref. [14, 19]" is as mentioned below:

1. Convert the image to grayscale image, if not.

2. Apply first level wavelet decomposition on skewed image. The image gets divided into four frequency bands i.e. LL1 (Approximation Level), LH1 (Horizontal Level), HL1 (Vertical Level), HH1 (Diagonal Level).

3. Apply second level wavelet decomposition to reduce skewed image further. Approximation level (LL1) gets divided into frequency bands LL2, LH2, HL2 and HH2.

4. Consider only horizontal 2nd level information (LH2) to find a skew angle for English typed documents. Make use of vertical and horizontal details at both levels, i.e. LH1, HL1, LH2, HL2 for scanned document images and for typed documents in other languages, i.e. Gujarati, Hindi, Urdu etc., horizontal and vertical details at second level i.e. LH2 and HL2 are considered.

5. Convert decomposed image matrix into a square matrix. Binarize it by setting a suitable threshold value.

6. Find the second order central moments of decomposed image, which is (LH2) to find a skew angle for English typed documents. If skew angle is $>45$, then add 90 .

7. In case of scanned documents, find a skew angle for LH1, HL1, LH2 and HL2 i.e. $\varnothing 1, \varnothing 2, \varnothing 3$,

$\varnothing 4$ by repeating the procedure of computing central moments. Consider absolute skew angle values and compute mean to find a final skew angle.

8. For typed documents in other languages, find skew angle for LH2, HL2 i.e. $\varnothing 1, \varnothing 2$. Find mean to find an accurate skew angle.

Advantages of Moments with wavelet transform

- In this method, the size of the original image is reduced, which improves time complexity. Simple 
and better than PCA with wavelet decomposition method

- Well suited for large size images.

Disadvantages of Moments with wavelet transform

- This method doesn't work with image documents.

\section{COMPARATIVE STUDY AND EXPERIMENTAL SETUP}

In order to analyze the performance, experiments have been carried out on a dataset of more than 150 images. Each image is tested 10 times to calculate the mean time and the mean skew angle. All these methods have been tested for skew angles varying from 0 to 90 degrees clockwise and anti-clockwise. The system used to implement skew detection methods has the following configuration: Intel ${ }^{\circledR}$ Core TM i7 CPU@ $2.67 \mathrm{GHz}, 2$ GB RAM. All these methods, namely Hough transform, Radon transforms, Principal Component Analysis (PCA), PCA with Wavelet transform and Moments with Wavelet transform to find skew distortion have been implemented using MATLAB version 10. Images of different sizes are created using Paint Shop Pro X5. Images used are tiff images only. The following methods, namely Hough transform, Radon transforms, Principal Component Analysis (PCA), PCA and Wavelet Transform and Moments with Wavelet transform have been implemented and comparative study has been carried out to find out the best method in terms of accuracy and efficiency. Different categories of document images used for testing are mentioned below:

1) Category 1: Scanned document images containing handwritten text/machine printed text of different languages (Fig. 1.1, 1.2, 1.3).

2) Category 2: Document images containing pictures (Fig. 2.1, 2.1)

3) Category 3: Scanned document images containing pictures (Fig. 3.1, 3.2).

4) Category 4: Machine printed images containing text of different fonts (Fig. 4.1, 4.2).

5) Category 5: Machine printed document images containing text and pictures (Fig. 5).

6) Category 6: Scanned document images containing text and pictures (Fig. 6).

7) Category 7: Mixed Scanned document (Fig. 7).

\section{A. Analysis of results on Category 1 images}

Fig. 1.1 shows scanned Urdu document image of size $256 \times 256$ pixels with resolution 300 pixels/inch of Category1. Fig 1.2 shows scanned Gujarati document image of size 256 × 256 pixels with resolution 300 pixels/inch of category 1 . Fig 1.3 shows scanned \& faded English document image written with pencil of size $419 x$ 185 pixels with resolution 150 pixels/inch of category 1 . From Table 1, it can be observed that in terms of time complexity, Radon transform is the fastest method with smaller document size for this category. The PCA method works with scanned document, but doesn't work with all languages, whereas Hough transform, Radon transform works with all languages and Moments with wavelet transform works with most of the languages. From Table 2 , it can be concluded that in terms of accuracy, Hough transform gives accurate results.

\section{B. Analysis of results on Category 2 images}

Fig. 2.1 shows a picture document image of size $181 \times 181$ pixels with resolution 78.74 pixels/inch of Category 2. Fig. 2.2 shows beans picture document image of size $91 \times 91$ pixels with resolution 28.35 pixels/inch of category 2. From Table 1, it can be observed that PCA, PCA with Wavelet transform and Moments with wavelet transform methods doesn't work with document containing picture images and Radon transform is the fastest method for this category. Accuracy is same for Hough and Radon transform as shown in Table 2.

\section{Analysis of results on Category 3 images}

Fig. 3.1 shows scanned picture document image of size $64 \times 64$ pixels with resolution 300 pixels/inch of category 3. Fig 3.2 shows scanned picture document image of size 196 x 210 pixels with resolution 300 pixels/inch of Category 3. As per Table 1, Radon transform is the fastest method as compared to other methods.

\section{Analysis of results on Category 4 images}

Fig. 4.1 shows machine printed document image of size $128 \times 128$ pixels with resolution 78.74 pixels/inch of Category 4. Fig 4.2 shows machine printed mixed font document image of size 64 x 64 pixels with resolution 96 pixels/inch of Category 4. After a Radon transforms, time taken by Moments with wavelet transform is minimum as per Table 1 if the image size is small. In terms of accuracy, Hough transform is more accurate as shown in Table 2. When the size of the same image is large i.e. $1024 X 1024$ pixels, then the time complexity of Moments with Wavelet transform method is better than other methods. It is thus fastest for such a case. PCA with Wavelet transform method is more accurate than PCA. Also, it takes less time than Hough transform, Radon Transform and PCA method, in case of large image size.

\section{E. Analysis of results on Category 5 images}

Fig. 5 shows machine printed text and picture document image of size $64 \times 64$ pixels with resolution 300 pixels/inch of Category 5. As mentioned earlier, it can be observed that PCA, PCA with Wavelet transform and Moments with Wavelet transform methods does not work with a document containing text and picture images, whereas Radon transform is the fastest method as per Table 1 but Hough transform gives more accurate results as shown in Table 2 .

\section{F. Analysis of results on Category 6 images}

Fig. 6 shows scanned text and picture document image of size $350 \times 350$ pixels with resolution 300 pixels/inch of Category 6. As mentioned earlier, it can be found PCA, PCA with Wavelet transform and Moments with Wavelet transform methods doesn't work with documents 
containing scanned text and picture images, whereas Radon transform is the fastest method as per Table 1 but Hough transform give more accurate results as shown in Table 2.

\section{G. Analysis of results on Category 7 images}

Fig. 7 shows scanned handwritten and machine text and picture document image of size 412 x 412 pixels with resolution 300 pixels/inch of Category 7 . It can be seen from Table 1 that Radon transform is the fastest method.

\section{CONCLUSION}

From the above experimental analysis, the following conclusions can be drawn:-

1. Radon transform is the fastest method for a scanned document image containing handwrittentext/machine-printed text in different languages, particularly in the case of small sized document. It works well and fast for document images containing pictures, scanned document images containing pictures, machine printed document images containing text and pictures, scanned document images containing text and pictures and mixed scanned document. It works with documents of all languages and documents with other kind of distortions (such as blurring) too.

2. Hough transform method determines the accurate skew angle, but it takes more time. Like Radon, it also works with documents in all languages and distorted documents too.

3. The PCA method doesn't work with picture related and all language documents but the speed is faster than Hough transform for scanned and machine printed document.

4. Moments with Wavelet transform is the fastest method in case of machine printed images containing text of different fonts, if the size of document is very large.

5. PCA with Wavelet transform method is faster and gives a more accurate angle than PCA method.

\section{REFERENCES}

[1] Chandan Singh, Nitin Bhatia and Amandeep Kaur, "Hough transform based fast skew detection and accurate skew correction methods," Pattern Recognition, vol. 41, pp.3528-3546, December 2008.

[2] N .Nandini, K. Srikanta Murthy, and G. Hemantha Kumar, "Estimation of skew Angle in binary document images using Hough transform," Journal of World Academy of Science, Engineering and Technology, vol. 32, pp. 50-55, August 2008.

[3] A.Amin and S.Fischer, "A document skew detection method using the Hough transform," Pattern Analysis and Applications, vol. 3, pp.243-253, September 2000.

[4] Stuart C.Hinds, James L.Fisher and Donald P.D'Amato, "A Document skew Detection Method Using Run Length Encoding and the Hough Transform," Proceedings of the Tenth International Conference on Pattern Recognition, pp.209 - 213, June 1990.
[5] S.N.Srihari and V.Govindraju, "Analysis of textual images using Hough transform," Journal of Machine Vision and Applications, vol. 2, pp.141-153, 1989.

[6] V.N.Manjunath Aradhya, G.Hemantha Kumar and P.Shivakumara, "Skew detection technique for binary document images based on Hough transform," International Journal of Information Technology, vol. 3, No. 3, pp. 194-200, 2006.

[7] R.O. Duda, P.E. Hart, "Use of the Hough transformation to detect lines and curves in picture", Magazine Communications of the ACM, pp. 11-15, vol. 15, No. 1, 1972.

[8] Gonzalez R.C, Woods, R.E., "Digital Image Processing", 3rd Edition, Prentice Hall, 2008.

[9] Rajiv Kapoor, Deepak Bagai and T.S.Kamal, "A new algorithm for skew detection and correction", Pattern Recognition Letters, vol. 25, pp.1215-1229, 2004.

[10] U.Pal and B.B.Chaudhuri ,"An improved document skew angle estimation technique", Pattern Recognition Letters, vol. 25, pp.1215-1229,2004.

[11] P.Toft, "The Radon transform-Theory and Implementation", Ph.D. thesis, Department of Mathematical Modeling, Technical University of Denmark, 1996.

[12] T.Steinherz, N. Intrator, E.Rivlin, "Skew detection via principal components analysis", Proceedings of the International Conference on Document Analysis and Recognition, pp. 153-156, 1999.

[13] N. D. Modi, C.K. Modi, C.N. Paunwala ,S. Patnaik, "Skew correction for vehicle license plates using principal component of Harris Corner Feature", Proceedings of the IEEE International Conference on Communication Systems and Network Technologies, pp. 339-343, 2011.

[14] S.Mehta, E. Walia, and M. Dutta, "A new fast approach for Skew Estimation using Moments and Wavelet Transform", International Conference on Image Processing Theory, Tools and Applications, pp. 221-226, 14-18 October 2014.

[15] C.N.Paunwala, S. Patnaik, and M. Chaudhary, "An efficient skew detection of license plate images based on wavelet transform and principal component analysis," in Proceedings of the IEEE International Conference on Signal and Image Processing, pp. 17-22, 2010.

[16] L.Shutao, S.Qinghua, and S.Jun, "Skew detection using wavelet decomposition and projection profile analysis," Pattern Recognition Letters, vol. 28, Issue 5, pp.555-562, April 2007.

[17] Bishakha Jain and Mrinaljit Borah, "A comparison paper on skew detection of scanned document images based on horizontal and vertical projection profile analysis," International Journal of Scientific and Research Publications, vol. 4, Issue 6, June 2014.

[18] D.Brodic, C.A.Maluckov, and L.Peng, "Estimation of the text skew in the old Printed documents," International Journal of Computer Communication, vol. 8, No. 5, pp. 673-680, Oct 2013.

[19] Shivakumara, G.Hemantha Kumar , and H.S.Varsha, "A new moments based skew estimation technique using pixels in the word for binary document images," Proceedings of the Eight IEEE International Conference on Document Analysis and Recognition, pp. 151-156, vol. 1, August 2005.

[20] Mandip Kaur and Simpel Jindal, "An integrated skew detection and correction using fast Fourier transform and DCT," International Journal of Scientific and Technology, vol. 2, pp. 164-169, December 2013.

[21] Deepak Kumar and Dalwinder Singh, "Modified approach 
[22] of Hough transform for skew detection and correction in document images," International Journal of Research in Computer Science, vol. 2, Issue 3, pp. 37-40, 2012.

[23] Sepideh Barekat Rezaei, Abdolhossein Sarrafzadeh and Jamshid Shanbehzadeh, "Skew detection of scanned document images," Proceedings of the International MultiConference of Engineers and Computer Scientists, vol. I, pp. 1-6, March 2013.

[24] Khalil Ibrahim Alsaif and Montaha Tariq Alsarraj, "New technique for skew angle of text in image document," International Journal of Information Technology and Business Management, vol.16, No.1, pp. 102-110, August 2013.

[25] H.Yan, "Skew correction of document images using interline cross-correlation," Journal of Graphic Models and Image Processing, vol. 55, pp. 538-543, November 1993.

[26] A.K. Das, B. Chanda, "A fast algorithm for skew detection of document images using morphology", International Journal on Document Analysis and Recognition, vol. 4, pp. 109-114, 2001.

[27] Ruby Singh, Ramandeep Kaur, "Improved skew detection and correction approach using Discrete Fourier algorithm", International Journal of soft computing and Engineering, vol. 3, Issue 4, pp. 5-7, September 2013.

\section{Authors' Profiles}

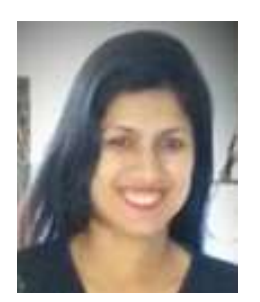

Sunita Mehta completed her Bachelor of Engineering in Computer Science in the year 1994 from Dharwad University, India, Masters in Information Technology from Punjabi University, Patiala, India in 2004 and pursuing Ph.D. (Computer Engineering) from Panjab University, Chandigarh, India. After completion of her Bachelor degree, she joined as Lecturer in Technical Education Department, Haryana, India. From 1998, she is serving as Lecturer in Computer Engineering at Chandigarh College of Engineering and Technology, U.T. Chandigarh Administration. She is presently working as officiating HOD of Electronics \& Communication Engineering and Computer Engineering. Her academic achievements include an award in graduation. Her research interest includes Digital Image Processing. She has presented research papers in national and international Conferences and published paper in International Journal.

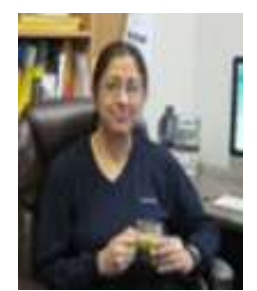

Dr. Ekta Walia received her Bachelors degree in Computer Science from Kurukshetra University, India and Masters in Computer Applications as well as Ph.D. (Computer Science) from Punjabi University, Patiala, India respectively. After starting her professional career as a software consultant with DCM DataSystems, New Delhi, India, in 1998, she served as Lecturer and Senior Lecturer in the National Institute of Technical Teachers Training and Research (NITTTR), Chandigarh, India for 07 years, where she was primarily involved in conducting short term courses for teachers of various engineering colleges and conduct of research. In 2011, she joined the Department of Computer Science in South Asian University, New Delhi, India, where she has been serving as Associate Professor and
Chairperson till May 2014. She joined the Department of Computer Science, University of Saskatchewan to work as Professional research associate in DADAISM project in June 2014. Her academic achievements include University Gold medals in Graduation as well as in Post-Graduation. Her research interests include 3D Rendering, Digital Image Watermarking, Content Based Image Retrieval and Face Recognition using orthogonal radial moments, in particular. She has a number of international journal and conference publications in these areas. She has been on the $\square$ reviewing board of reputed image processing journals and conferences. She has also chaired sessions in International Conferences of repute.

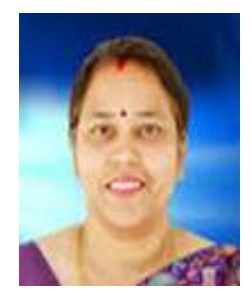

Dr. Maitreyee Dutta completed her Bachelor of Engineering in Electronics and Telecommunication in the year 1992 from Guwahati University, India, M.E in Electronics and Telecommunication and Ph.D. from Panjab University, Chandigarh, India in 1998 and 2007 respectively. She started her career as Lecturer in Electronics and Telecommunication Department from 1993-1994 at Assam Engineering College, Guwahati, India. She served as Project Associate in Computer Science Department at Technical Teachers Training Institute, Chandigarh, India from 1995-1996. After that, she was appointed as senior faculty at Aptech Computer Education, Panchkula, India from 1998-2001. She worked as Lecturer (Computer Science) from 2001 to May 2002, System Programmer from Jun 2002 to Jan 2006, Assistant Professor and Head, Computer Science Department from Feb 2006 and worked as Associate Professor from 2009 to 2014 at National Institute of Technical Teachers Training and Research, Chandigarh, India. She is presently working as Professor since 2014 at National Institute of Technical Teachers Training and Research, Chandigarh. During her service, she has guided nearly 60 thesis for M.E course and actively involved in research work. Her academic achievements include University Gold medal during graduation as well as received a national scholarship during B.E and secondary level. She is a reviewer of Journal of Multi Disciplinary Engineering Technologies. She has presented 26 papers in International Journal and presented 49 papers in national and international conferences. Her area of research includes Digital Signal Processing, Digital Image Processing and Cloud Computing.

Manuscript received: June 25, 2015; revised July 18, 2015; accepted August 21, 2015.

How to cite this paper: Sunita Mehta, Ekta Walia, Maitreyee Dutta,"Time and Accuracy Analysis of Skew Detection Methods for Document Images", International Journal of Information Technology and Computer Science(IJITCS), vol.7, no.11, pp.43-54, 2015. DOI: 10.5815/ijitcs.2015.11.06 


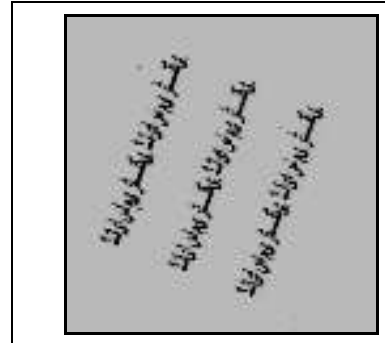

(a)

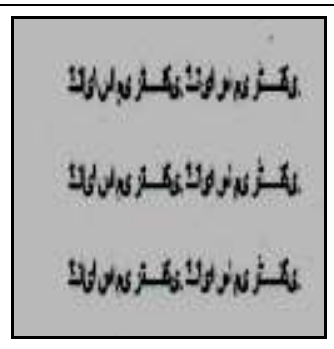

(b)

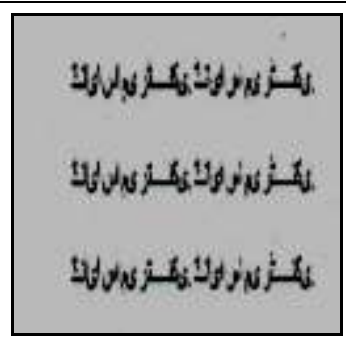

(c)

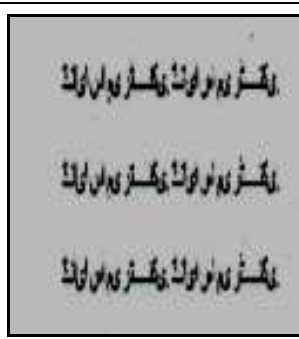

(d)

Fig.1.1. Category 1 Sample skewed and skew corrected Urdu language scanned image. (a) Scanned Urdu image distorted by $70^{\circ}$ (b) Image corrected with Radon Transform (c) Image corrected with Hough Transform (d) Image corrected with Moments with Wavelet Transform

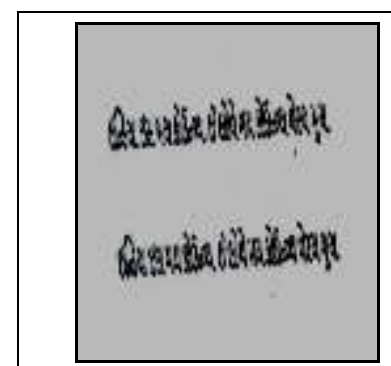

(a)

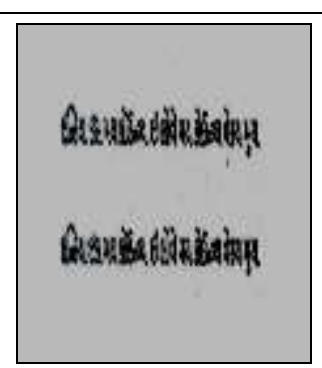

(b)

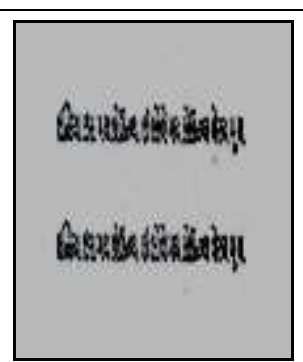

(c)

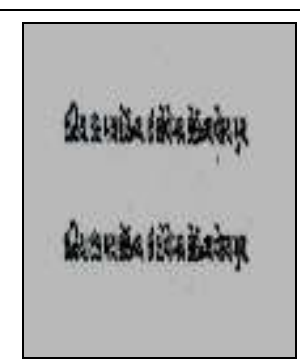

(d)

Fig.1.2. Category 1 Sample skewed and skew corrected Gujarati language scanned image. (a) Scanned Gujarati image distorted by $5^{0}$ (b) Image corrected with Radon Transform (c) Image corrected with Hough Transform (d) Image corrected with Moments and Wavelet Transform

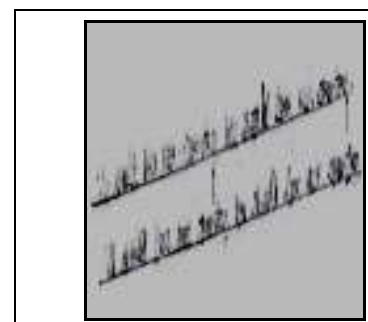

(a)

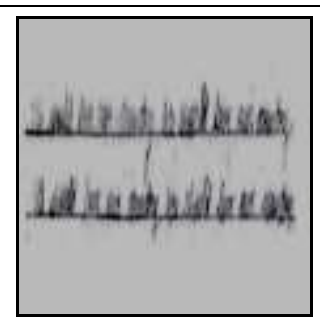

(b)

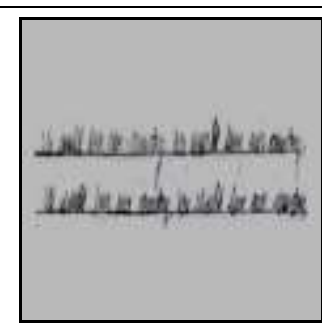

(c)

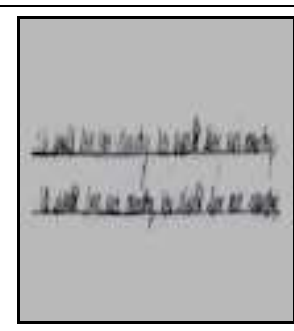

(d)

Fig.1.3. Category 1 Sample skewed and skew corrected English Language scanned image (faded image chosen to experiment). (a) Scanned English faded image distorted by $10^{\circ}$ (b) Image corrected with PCA (c) Image corrected with Hough Transform (d) Image corrected with Radon Transform

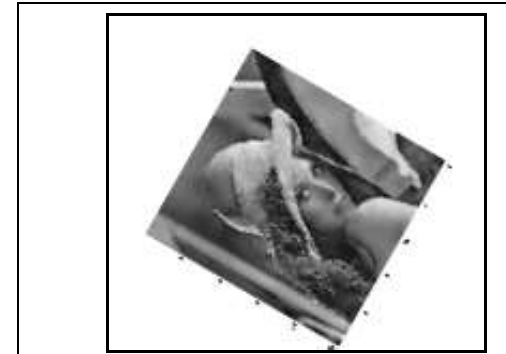

(a)

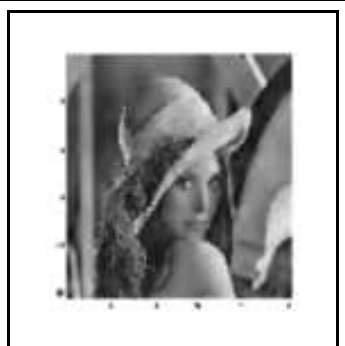

(b)

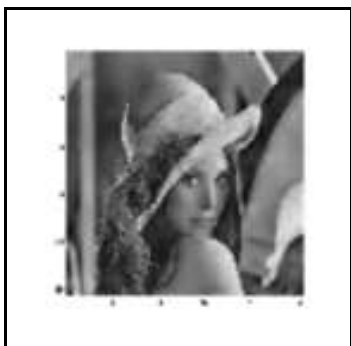

(c)

Fig.2.1. Category 2 Sample skewed and skew corrected picture Image (a) Picture image distorted by $60^{\circ}$ (b) Image corrected with Radon Transform (c) Image corrected with Hough Transform 


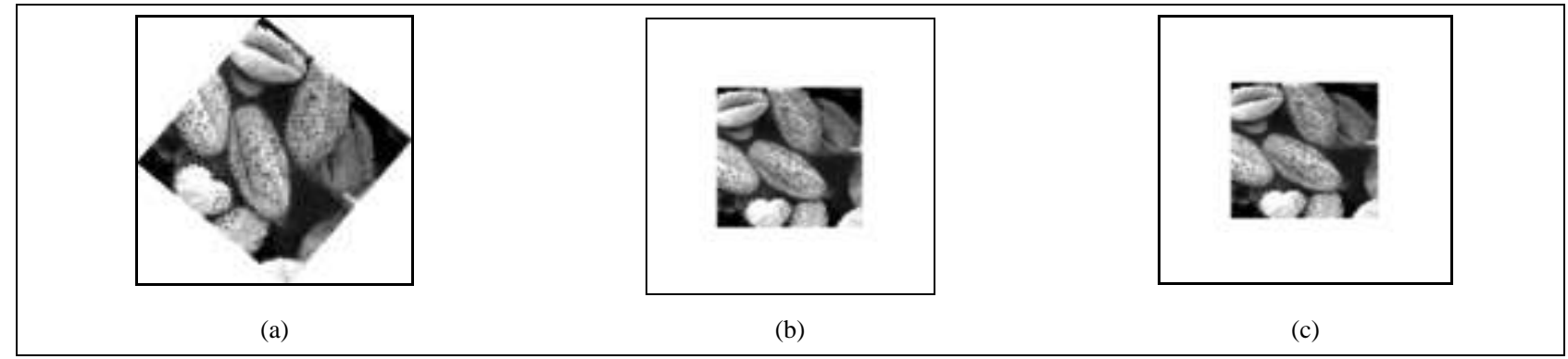

Fig.2.2. Category 2 Sample skewed and skew corrected picture Image (a) Picture image distorted by $40^{\circ}$ (b) Image corrected with Hough Transform (c) Image corrected with Radon Transform

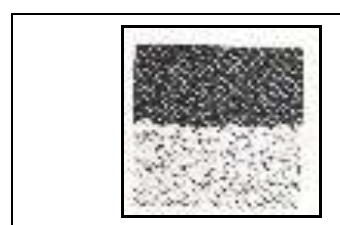

(a)

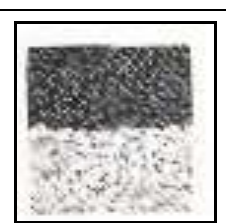

(b)

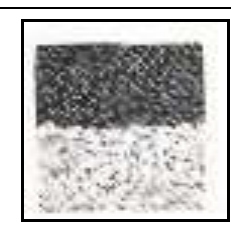

(c)

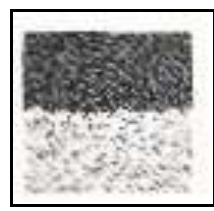

(d)

Fig.3.1. Category 3 Sample skewed and skew corrected scanned Image (a) Scanned Image of $2^{\circ}$ distortion (b) Image corrected with Radon Transform (c) Image corrected with Hough Transform (d) Image corrected with PCA

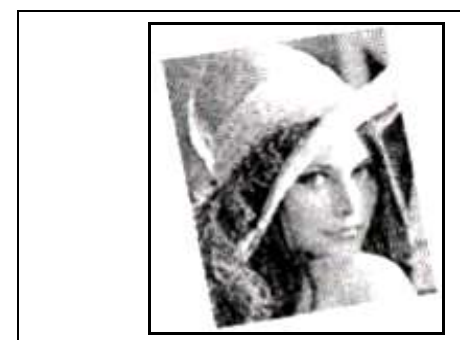

(a)

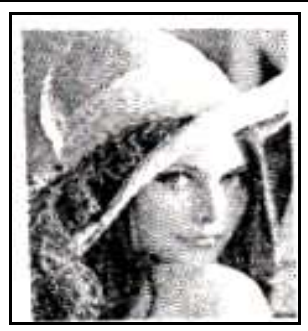

(b)

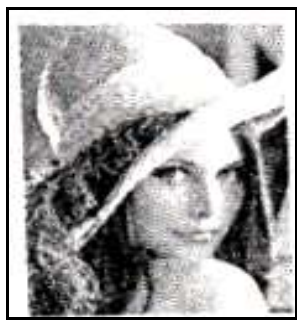

(c)

Fig.3.2. Category 3 Sample skewed and skew corrected scanned picture Image (a) picture image distorted by $9^{0}$ (b) Image corrected with Radon Transform (c) Image corrected with Hough Transform

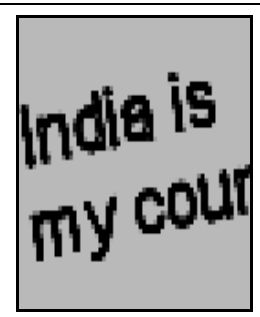

(a)

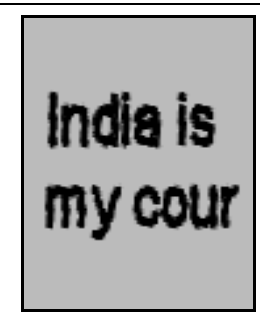

(b)

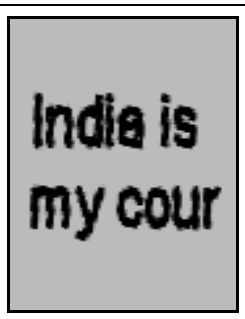

(c)

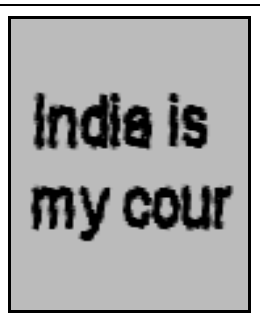

(d)

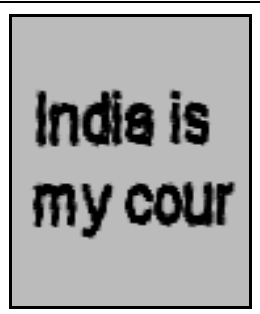

(e)

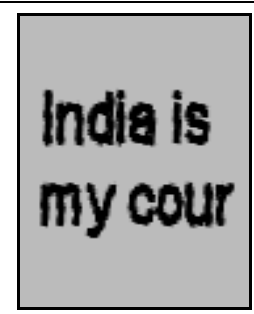

(f)

Fig.4.1. Category 4 Sample skewed and skew corrected machine printed Image (a) Machine Printed Image of $10^{\circ}$ distortion (b) Image corrected with PCA with Wavelet Transform (c) Image corrected with PCA (d) Image corrected with Radon Transform (e) Image corrected with Hough Transform (f) Image corrected with Moments with Wavelet Transform

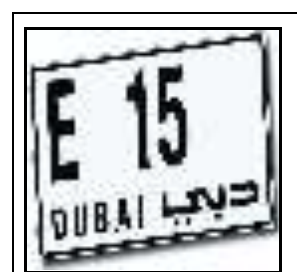

(a)

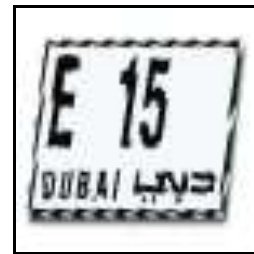

(b)

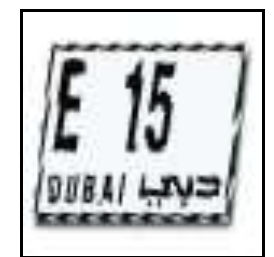

(c)

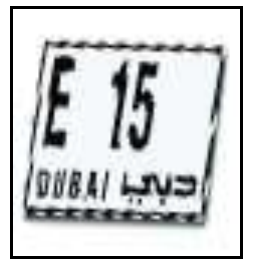

(d)

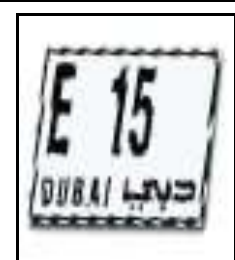

(e)

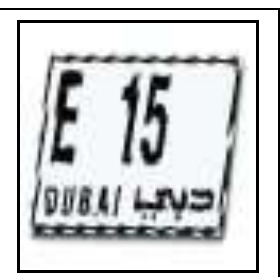

(f)

Fig.4.2. Category 4 Sample skewed and skew corrected machine printed mixed font Image (a) Image of car number plate of $9^{0}$ distortion (b) Image corrected with Radon Transform (c) Image corrected with Hough Transform (d) Image corrected with PCA (e) Image corrected with PCA with Wavelet (f) Image corrected with Moments with Wavelet Transform 


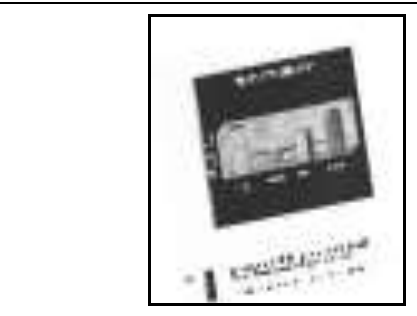

(a)

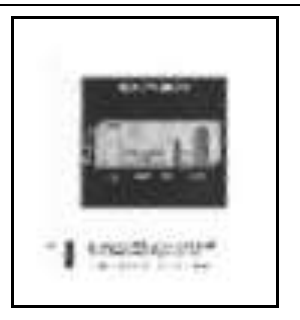

(b)

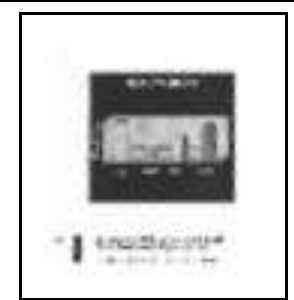

(c)

Fig.5. Category 5 Sample skewed and skew corrected mixed text and picture Image (a) Text and Picture image distorted by $10^{\circ}$ (b) Image corrected with Radon Transform (c) Image corrected with Hough Transform

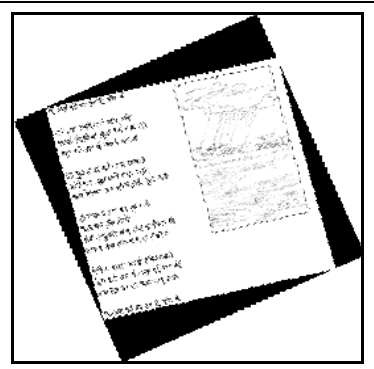

(a)

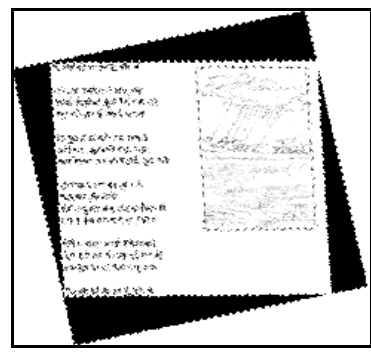

(b)

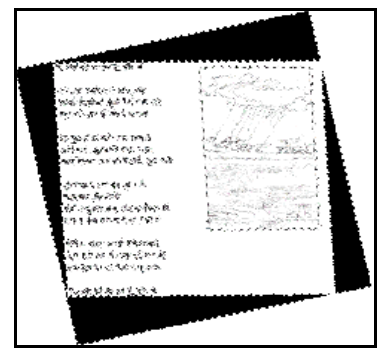

(c)

Fig.6. Category 6 Sample skewed and skew corrected scanned mixed text and picture Image (a) Scanned Text and Picture image distorted by $12^{\circ}$ (b) Image corrected with Radon Transform (c) Image corrected with Hough Transform

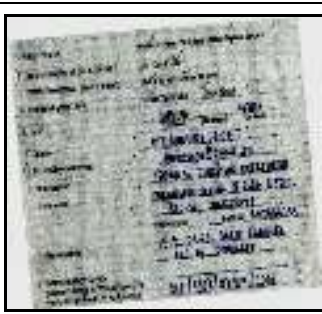

(a)

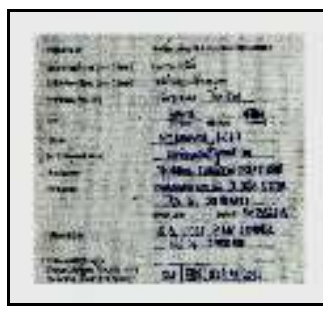

(b)

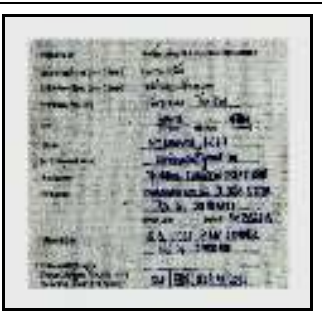

(c)

Fig.7. Category 7 Sample skewed and skew corrected machine and hand mixed scanned Image (a) Machine Printed and Hand mixed image distorted by $5^{\circ}$ (b) Image corrected with Radon Transform (c) Image corrected with Hough Transform

Table 1. Comparison of Hough transform, Radon transform, PCA, PCA with Wavelet transform and Moments with Wavelet transform in terms of mean time required for skew detection

\begin{tabular}{|c|c|c|c|c|c|c|}
\hline Name of Image & $\begin{array}{c}\text { Actual } \\
\text { Skew } \\
\text { Angle } \\
\text { (in degree) }\end{array}$ & $\begin{array}{l}\text { Mean Time taken } \\
\text { using Hough } \\
\text { Transform (in } \\
\text { secs) }\end{array}$ & $\begin{array}{l}\text { Mean Time taken } \\
\text { using Radon } \\
\text { Transform } \\
\text { (in secs) }\end{array}$ & $\begin{array}{c}\text { Mean Time } \\
\text { taken using PCA } \\
\text { (in secs) }\end{array}$ & $\begin{array}{c}\text { Mean Time } \\
\text { taken } \\
\text { Using PCA } \\
\text { with WT } \\
\text { (in secs) }\end{array}$ & $\begin{array}{l}\text { Mean Time taken } \\
\text { Using moments } \\
\text { with WT } \\
\text { (in secs) }\end{array}$ \\
\hline Fig. 1.1 & 70 & 0.41 & 0.07 & - & - & 0.262 \\
\hline Fig. 1.2 & 5 & 0.35 & 0.07 & - & - & 0.21 \\
\hline Fig. 1.3 & 10 & 0.81 & 0.1 & 0.25 & - & - \\
\hline Fig. 2.1 & 60 & 0.38 & 0.18 & - & - & - \\
\hline Fig. 2.2 & 40 & 0.34 & 0.01 & - & - & - \\
\hline Fig. 3.1 & 2 & 0.35 & 0.005 & - & - & - \\
\hline Fig. 3.2 & 9 & 0.80 & 0.13 & - & - & - \\
\hline $\begin{array}{c}\text { Fig. 4.1(image of } \\
128 X 128 \text { pels) }\end{array}$ & 10 & 0.46 & 0.01 & 0.02 & 0.018 & 0.0175 \\
\hline $\begin{array}{l}\text { Fig. 4.1(image of } \\
1024 \text { X1024 pels) }\end{array}$ & 10 & 1.47 & 1.05 & 1.1 & 0.70 & 0.65 \\
\hline Fig. 4.2 & 9 & 0.34 & 0.034 & 0.06 & 0.055 & 0.049 \\
\hline Fig. 5 & 10 & 0.76 & 0.014 & - & - & - \\
\hline Fig. 6 & 12 & 0.84 & 0.15 & - & - & - \\
\hline Fig. 7 & 5 & 0.90 & 0.30 & - & - & - \\
\hline
\end{tabular}


Table 2. Comparison of Hough transform, Radon transform, PCA, PCA with Wavelet transform and Moments with Wavelet transform to calculate Skew Angle

\begin{tabular}{|c|c|c|c|c|c|c|c|c|c|c|c|}
\hline $\begin{array}{l}\text { Name of } \\
\text { Image }\end{array}$ & $\begin{array}{c}\text { Actual } \\
\text { Skew } \\
\text { Angle } \\
\text { (in } \\
\text { degree) }\end{array}$ & $\begin{array}{c}\text { Skew } \\
\text { Angle } \\
\text { using } \\
\text { Hough } \\
\text { Transfor } \\
\text { m } \\
\text { (in } \\
\text { degree) }\end{array}$ & Error & $\begin{array}{c}\text { Skew } \\
\text { Angle } \\
\text { using } \\
\text { Radon } \\
\text { Transfor } \\
\text { m } \\
\text { (in } \\
\text { degree) }\end{array}$ & Error & $\begin{array}{c}\text { Skew } \\
\text { Angle } \\
\text { using } \\
\text { PCA } \\
\text { (in } \\
\text { degree) }\end{array}$ & Error & $\begin{array}{c}\text { Skew } \\
\text { Angle } \\
\text { using } \\
\text { PCA } \\
\text { With } \\
\text { WD(in } \\
\text { degree) }\end{array}$ & Error & $\begin{array}{c}\text { Skew } \\
\text { Angle } \\
\text { using } \\
\text { moment } \\
\text { With } \\
\text { WD(in } \\
\text { degree) }\end{array}$ & Error \\
\hline Fig. 1.1 & 70 & 70.04 & 0.04 & 70.04 & 0.04 & - & - & - & - & -70.25 & 0.25 \\
\hline Fig. 1.2 & 5 & 5.04 & 0.04 & -4.95 & 0.05 & - & - & - & - & -5.2 & 0.2 \\
\hline Fig. 1.3 & 10 & 10.0 & 0 & 10.04 & 0.04 & -10.3 & 0.3 & - & - & - & - \\
\hline Fig. 2.1 & 60 & -60.04 & 0.04 & -59.96 & 0.04 & - & - & - & - & - & - \\
\hline Fig. 2.2 & 40 & 40.04 & 0.04 & 40.04 & 0.04 & - & - & - & - & - & - \\
\hline Fig. 3.1 & 2 & -2.04 & 0.04 & 2.04 & 0.04 & - & - & - & - & - & - \\
\hline Fig. 3.2 & 9 & 9.04 & 0.04 & 9.06 & 0.06 & - & - & - & - & - & - \\
\hline Fig. 4.1 & 10 & 10.04 & 0.04 & -9.95 & 0.05 & 10.28 & 0.28 & 9.75 & 0.25 & -10.20 & 0.20 \\
\hline Fig. 4.2 & 9 & 9.04 & 0.04 & 8.95 & 0.05 & 9.73 & 0.73 & 9.66 & 0.66 & -9.31 & 0.31 \\
\hline Fig. 5 & 10 & 10.04 & 0.04 & -9.95 & 0.05 & - & - & - & - & - & - \\
\hline Fig. 6 & 12 & 12.04 & 0.04 & 11.94 & 0.06 & - & - & - & - & - & - \\
\hline Fig. 7 & 5 & -5.04 & 0.04 & 5.04 & 0.04 & - & - & - & - & - & - \\
\hline
\end{tabular}

